\title{
Superweak-like models of CP violation
}

\author{
L. Lavoura \\ Department of Physics, Carnegie-Mellon University, \\ Pittsburgh, Pennsylvania 15213, U.S.A.
}

June 19, 1992

\begin{abstract}
I put forward a two-Higgs-doublet model in which CP violation is mediated only by the neutral Higgs bosons, via the mechanism of scalar-pseudoscalar mixing. In this model there is no CP violation in the exchange of either $W$ bosons or of charged Higgs bosons. The model is therefore an approximate realization of the superweak theory of CP violation. It has only two basic CP-violating quantities. I point out that other models of this kind, but with more than two Higgs doublets, may also be built.
\end{abstract}

\section{Introduction}

The simplest explanation of the CP violation observed in the neutral-kaon system is given by the superweak theory, which was suggested by Wolfenstein in 1964 [1]. From the modern point of view, that theory is purely phenomenological. It states that the only CP-odd terms in the Lagrangian are terms which change the quark flavor (for instance, the strangeness) by two units, having as a consequence that $C P$ violation occurs only in the mixing in the neutral-meson systems, and does not occur in the decay amplitudes. This theory explains perfectly the observed $\mathrm{CP}$ violation, because all of it can be attributed to one CP-violating parameter, $\epsilon$, in the mixing of the neutral kaons. The superweak theory would have to be discarded if a nonzero value for the parameter $\epsilon^{\prime}$ were measured; it would also have to be discarded if different CP asymmetries in different decay modes of the neutral- $B$-meson system were observed [2]; there is also the possibility of observing direct CP violation in CP-conjugated decays of charged $B$ mesons. Even if the superweak theory would finally have to be discarded, the possible existence of a contribution to CP violation from an interaction of the superweak type would lead to interesting consequences, like $C P$ asymmetries in the neutral- $B$-meson decays at wild variance with the predictions of the standard model [3].

Though the superweak theory has been very resistant to the experimental test, it is difficult to integrate that phenomenological theory into the modern gauge models of the fundamental interactions. It is known that the standard model may, for a top-quark mass of about $200 \mathrm{GeV}$, simulate a superweak theory, in that it predicts 
a very small value for $\epsilon^{\prime}[4]$; but that simulation occurs only in what concerns that particular parameter, and it is moreover in no way fundamental, being only the result of the accidental cancellation of various contributions to $\epsilon^{\prime}$. Also, interactions in which C.P is violated via the exchange of neutral Higgs particles with flavor-changing Yukawa couplings, are of superweak nature; but it is not correct to assert that a model in which such interactions arise realizes the superweak theory, because usually those models also include CP violation mediated by charged Higgs particles and/or by the $W$ boson [5] (Kobayashi-Maskawa CP violation [6]). Extra assumptions, like supposing that the charged Higgs bosons are very heavy, or that the phase in the CKM matrix [6] is very small. are then needed; else, the extra sources of $C P$ violation will both induce direct $\mathrm{CP}$ violation, and tend to destroy the predictive power of the model in what concerns CP violation.

The purpose of the present work is to study ways of building models in which CP violation occurs only via the flavor-changing exchange of neutral Higgs bosons, while there is no Kobayashi-Maskawa CP violation, and also no CP violation mediated by charged Higgs bosons. Such models will be realizations of the superweak theory, if one makes the reasonable assumption that the exchange of the neutral Higgs particles does not contribute significantly to the various decay amplitudes, which means that these are still dominated by the exchange of the $W$ boson, and are therefore real. I emphasize however that this assumption may not hold in these models. Furthermore, these models have contributions to quark dipole moments at the one-loop level, in contrast to what occurs in a genuine superweak theory. Anyway, I will rather loosely say models with the features above to be superweak-like. The models that I will present, in particular the one of section 3 , have the advantage of having the CP violation restricted to a few basic interactions, and parametrized by a relatively small number of quantities.

In section 2 I discuss some general features of a two-Higgs-doublet model with a softly-broken $Z_{2}$ symmetry. Most of the material in that section is known, and I write it down mainly in order to fix the notation. In section 3 I show that, if the $Z_{2}$ symmetry applies to the fermion sector of the theory in a particular way, one obtains a model with $\mathrm{CP}$ violation only in the scalar-pseudoscalar mixing. In section $4 \mathrm{I}$ show that similar models may be constructed with more than two Higgs doublets, with the help of larger discrete-symmetry groups. As an example of this, I give in section 5 a model with four Higgs doublets, which has the bonus of some predictive power in what concerns the form of the CKM matrix. The main conclusions are summarised in section 6 .

\section{The two-Higgs-doublet model}

I work in the context of a three-family $S U(2) \times U(1)$ model. In a weak basis, the three quark doublets are denoted by $q_{L}=\left(p_{L}, n_{L}\right)^{T}$, and the right-handed up-type and down-type quarks are denoted by $p_{R}$ and $n_{R}$, respectively. With two Higgs doublets $H_{1}$ and $H_{2}$, the Yukawa couplings are of the form

$$
\mathcal{L}_{1}=-\overline{q_{l}} H_{1} \Gamma_{1} n_{R}-\overline{q_{l}} H_{2} \Gamma_{2} n_{R}
$$




$$
\begin{aligned}
& -\overline{q_{L}}\left(i \sigma_{2} H_{1}^{*}\right) \Delta_{1} p_{R}-\overline{q_{L}}\left(i \sigma_{2} H_{2}^{*}\right) \Delta_{2} p_{R} \\
& + \text { h.c. }
\end{aligned}
$$

Because I want to avoid the presence of Kobayashi-Maskawa CP violation, I will consider models in which CP invariance holds at the Lagrangian level, but is spontaneously broken [ 7$]$. I therefore take the $3 \times 3$ matrices of Yukawa couplings $\Gamma_{1}, \Gamma_{2}, \Delta_{1}$ and $\Delta_{2}$ to be real.

The Higgs doublets are written as

$$
H_{1}=\left(\begin{array}{c}
\varphi_{1}^{+} \\
\frac{1}{\sqrt{2}}\left(v_{1}+\rho_{1}+i \eta_{1}\right)
\end{array}\right), \quad H_{2}=e^{\prime r}\left(\begin{array}{c}
\varphi_{2}^{+} \\
\frac{1}{\sqrt{2}}\left(v_{2}+\rho_{2}+i \eta_{2}\right)
\end{array}\right),
$$

where $v_{1} / \sqrt{2}$ and $e^{i t r} v_{2} / \sqrt{2}$ are the vacuum expectation values (vevs) of $H_{1}^{\prime \prime}$ and $H_{2}^{\prime \prime}$, respectively. There is no loss of generality in taking the vev of $H_{1}^{\prime \prime}$ to be real and positive. I re-define both the charged and the neutral components of these fields by means of the orthogonal matrix

$$
O=\left(\begin{array}{rr}
v_{1} / v & v_{2} / v \\
v_{2} / v & -v_{1} / v
\end{array}\right)
$$

in which $v:=\sqrt{v_{1}^{2}+v_{2}^{2}}=\left(\sqrt{2} G_{F}\right)^{-1 / 2}$, in the following way:

$$
\left(\begin{array}{c}
G^{+} \\
H^{+}
\end{array}\right)=O\left(\begin{array}{c}
\varphi_{1}^{+} \\
\varphi_{2}^{+}
\end{array}\right), \quad\left(\begin{array}{c}
G^{\prime \prime} \\
I
\end{array}\right)=O\left(\begin{array}{l}
\eta_{1} \\
\eta_{2}
\end{array}\right), \quad\left(\begin{array}{c}
H^{0} \\
R
\end{array}\right)=O\left(\begin{array}{l}
\rho_{1} \\
\rho_{2}
\end{array}\right) .
$$

$G^{+}$and $G^{\prime \prime}$ are the charged and neutral Goldstone bosons, respectively, which become the longitudinal components of the $W^{+}$and the $Z^{\cup}$, in the U-gauge.

The mass matrices of the quarks are bi-diagonalised, as usual, by $3 \times 3$ unitary matrices $U_{n L}, U_{n R}, U_{p L}$ and $U_{p R}$ :

$$
\begin{aligned}
U_{n l}^{\dagger}\left(\frac{v_{1}}{\sqrt{2}} \Gamma_{1}+\frac{v_{2}}{\sqrt{2}} e^{i \alpha x} \Gamma_{2}\right) U_{n R} & =D_{d}, \\
U_{p L}^{\dagger}\left(\frac{v_{1}}{\sqrt{2}} \Delta_{1}+\frac{v_{2}}{\sqrt{2}} e^{-i n} \Delta_{2}\right) U_{p R} & =D_{u},
\end{aligned}
$$

where $D_{d}$ and $D_{u}$ are the diagonal matrices of the masses of the down-type and up-type quarks, respectively. I define the two non-hermitian matrices $N_{d}$ and $N_{u}$ as

$$
\begin{aligned}
& N_{d}:=U_{n L}^{\dagger}\left(\frac{v_{2}}{\sqrt{2}} \Gamma_{1}-\frac{v_{1}}{\sqrt{2}} e^{i n} \Gamma_{2}\right) U_{n R}, \\
& N_{u}:=U_{p L}^{\dagger}\left(\frac{v_{2}}{\sqrt{2}} \Delta_{1}-\frac{v_{1}}{\sqrt{2}} e^{-u x} \Delta_{2}\right) U_{p R} .
\end{aligned}
$$

Then, the Yukawa interactions of $H^{+}, H^{\cup}, R$ and $I$ are given by

$$
\begin{aligned}
\mathcal{L}_{1}= & \ldots+\sqrt{2} \frac{H^{+}}{v} \bar{u}\left(N_{u}^{\dagger} V \gamma_{l}-V N_{d} \gamma_{R}\right) d+\sqrt{2} \frac{H^{-}}{v} \bar{d}\left(V^{\dagger} N_{u} \gamma_{R}-N_{d}^{\dagger} V^{\dagger} \gamma_{I}\right) u \\
& -\frac{H^{\prime}}{v}\left(\bar{u} D_{1} u+\bar{d} D_{d} d\right) \\
& -\frac{R}{v}\left[\bar{u}\left(N_{u} \gamma_{R}+N_{u}^{\dagger} \gamma_{L}\right) u+\bar{d}\left(N_{d} \gamma_{R}+N_{d}^{\dagger} \gamma_{L}\right) d\right] \\
& +i \frac{I}{v}\left[\bar{u}\left(N_{u} \gamma_{R}-N_{u}^{\dagger} \gamma_{L}\right) u-\bar{d}\left(N_{d} \gamma_{R}-N_{d}^{\dagger} \gamma_{L}\right) d\right] .
\end{aligned}
$$


Here. $V^{\prime}:=U_{n L}^{\dagger} U_{n l}$ is the CKM matrix, and $\gamma_{R . I .}:=\left(1 \pm \gamma_{5}\right) / 2 . u$ and $d$ are the column matrices of the physical up- and down-type quark fields, respectively. $H^{\prime \prime}$ couples as the standard-model Higgs field, but in the two-Higgs-doublet model it mixes with $R$ and $I$, the couplings of which are not flavor-diagonal. The couplings of the Goldstone bosons $G^{+}$and $G^{\prime \prime}$ are of course the same as in the standard model.

Notice that, from Eqs. 5 and 6, one has

$$
N_{d}=\frac{v_{2}}{v_{1}} D_{d}-\frac{v_{2}}{\sqrt{2}}\left(\frac{v_{2}}{v_{1}}+\frac{v_{1}}{v_{2}}\right) U_{n l}^{\dagger} e^{i \prime \prime} \Gamma_{2} U_{n / R} .
$$

I will use this result later.

The most general Higgs potential for the two-Higgs-doublet model was written by T. D. Lee in his pioneering work on spontaneous CP violation [7]. It has since become customary to consider a version of the model in which a discrete symmetry $S$

$$
S: H_{1} \rightarrow H_{1}, H_{2} \rightarrow-H_{2}
$$

(I do not write yet the transformation properties of the quark fields) is introduced, usually with the purpose of avoiding the presence of flavor-changing neutral interactions (FCNI). In my model FCNI are wanted, because they will constitute the superweak interaction. However, in order to obtain real $m$ iss matrices, I will still need the discrete symmetry $S$, though implemented in a different way in the quark sector. On the other hand, there may be various motivations [8] for introducing a term in the Higgs potential which breaks $S$ softly; in the present case I need that term in order to obtain the possibility of CP being spontareously broken. The Higgs potential is

$$
\begin{aligned}
\mathcal{L}_{H}= & \mu_{1} H_{1}^{\dagger} H_{1}+\mu_{2} H_{2}^{\dagger} H_{2}+\mu_{3}\left(H_{1}^{\dagger} H_{2}+\text { h.c. }\right)+a_{1}\left(H_{1}^{\dagger} H_{1}\right)^{2}+a_{2}\left(H_{2}^{\dagger} H_{2}\right)^{2} \\
& +b\left(H_{1}^{\dagger} H_{1}\right)\left(H_{2}^{\dagger} H_{2}\right)+c\left(H_{1}^{\dagger} H_{2}\right)\left(H_{2}^{\dagger} H_{1}\right)+d\left[\left(H_{1}^{\dagger} H_{2}\right)^{2}+\text { h.c. }\right] .
\end{aligned}
$$

The coefficient of the term which breaks $S$ softly, $\mu_{3}$, is assumed to be much smaller than the other two dimensional coefficients in the potential, $\mu_{1}$ and $\mu_{2}$. This assumption is natural, because the Lagrangian acquires an extra symmetry, $S$, when $\mu_{3}$ vanishes. The stationarity conditions are

$$
\begin{aligned}
2 \mu_{1} & =-2 a_{1} v_{1}^{2}-k v_{2}^{2}, \\
2 \mu_{2} & =-2 a_{2} v_{2}^{2}-k v_{1}^{2}, \\
\mu_{3} & =-2 d v_{1} v_{2} \cos \alpha,
\end{aligned}
$$

where $k:=b+c-2 d$. They fix $v_{1}, v_{2}$ and $\alpha$ as functions of $\mu_{1}, \mu_{2}$ and $\mu_{3}$. If all the dimensionless parameters in the Higgs potential are of the same order of magnitude, then the fact that $\mu_{3}$ is much smaller than $\mu_{1}$ and $\mu_{2}$ implies $\cos \alpha \ll 1$, and therefore the phase $\alpha$ is very close to $\pm \pi / 2$, which means that the CP violation via scalarpseudoscalar mixing is very small. This way of naturally suppressing CP violation was first suggested by Branco and Rebelo [9]. 
We readily obtain, from the potential in Eq. 10, and after using the stationarity conditions in Eqs. 11, the mass terms for the Higgs bosons:

$$
\mathcal{L}_{H}=\ldots+\frac{2 d-c}{2} v^{2} H^{-} H^{+}+\left(\begin{array}{lll}
H^{U} & R & I
\end{array}\right) \mathcal{M}\left(\begin{array}{l}
H^{\prime} \\
R \\
I
\end{array}\right),
$$

where the real and symmetric matrix $\mathcal{M}$ has

$$
\begin{aligned}
& \mathcal{M}_{11}=\frac{1}{v^{2}}\left[a_{1} v_{1}^{1}+a_{2} v_{2}^{1}+v_{1}^{2} v_{2}^{2}\left(k+4 d \cos ^{2} \alpha\right)\right] \\
& \mathcal{M}_{22}=\frac{1}{v^{2}}\left[d\left(v_{1}^{2}-v_{2}^{2}\right)^{2} \cos ^{2} \alpha+v_{1}^{2} v_{2}^{2}\left(a_{1}+a_{2}-k\right)\right] \\
& \mathcal{M}_{33}=d v^{2} \sin ^{2} \alpha \\
& \mathcal{M}_{12}=\frac{v_{1} v_{2}}{v^{2}}\left[a_{1} v_{1}^{2}-a_{2} v_{2}^{2}+\frac{v_{2}^{2}-v_{1}^{2}}{2}\left(k+4 d \cos ^{2} \alpha\right)\right] \\
& \mathcal{M}_{13}=d v_{1} v_{2} \sin (2 \alpha), \\
& \mathcal{M}_{23}=\frac{d}{2}\left(v_{2}^{2}-v_{1}^{2}\right) \sin (2 \alpha) .
\end{aligned}
$$

There are two non-trivial equations among the matrix elements of $\mathcal{M}$ :

$$
\begin{aligned}
\frac{\mathcal{M}_{23}}{\mathcal{M}_{13}} & =\frac{1}{2}\left(\frac{v_{2}}{v_{1}}-\frac{v_{1}}{v_{2}}\right), \\
\frac{\mathcal{M}_{1: 3}^{2}+\mathcal{M}_{2 \cdot 3}^{2}}{\mathcal{M}_{33}^{2}} & =\cot ^{2} \alpha .
\end{aligned}
$$

These relations are not very useful in practice, because in practical calculations the matrix elements of interest are the ones of $\mathcal{M}^{-1}$, and Eqs. 19 and 20 are more complicated when written in terms of these. But these equations tell us that there are only four independent mixed Higgs-boson propagators or, equivalently, matrix elements of $\mathcal{M}^{-1}$.

The mass matrix $\mathcal{M}$ is diagonalized by an orthogonal matrix $T$ :

$$
T^{T} \mathcal{M} T=\operatorname{diag}\left(\frac{m_{1}^{2}}{2}, \frac{m_{2}^{2}}{2}, \frac{m_{3}^{2}}{2}\right),
$$

where the three $m_{i}$ are the masses of the three real neutral Higgs fields $H_{i}$. It follows that

$$
\begin{aligned}
\sum_{i=1}^{3} \frac{2 T_{1} T_{31}}{m_{i}^{2}}=\left(\mathcal{M}^{-1}\right)_{13} & =\frac{2 \cot \alpha}{v^{2} v_{1} v_{2}} \frac{k v^{2}-2 a_{1} v_{1}^{2}-2 a_{2} v_{2}^{2}}{4 a_{1} a_{2}-k^{2}} \\
\sum_{i=1}^{3} \frac{2 T_{21} T_{31}}{m_{i}^{2}}=\left(\mathcal{M}^{-1}\right)_{23} & =\frac{4 \cot \alpha}{v^{2} v_{1}^{2} v_{2}^{2}} \frac{a_{1} v_{1}^{1}-a_{2} v_{2}^{1}}{4 a_{1} a_{2}-k^{2}}
\end{aligned}
$$

These matrix elements govern the mixing of the scalars $H^{0}$ and $R$, respectively, with the pseudoscalar $I$. In the model of the next section, all the CP-violating quantities 
are (in the point-interaction approximation for the neutral-Higgs-exchange diagrams) linear combinations of $\left(\mathcal{M}^{-1}\right)_{13}$ and $\left(\mathcal{M}^{-1}\right)_{23}$. They are therefore all proportional to $\cot \alpha \ll 1$, which fact gives a natural suppression of CP violation [9]. Notice that $\mathcal{M}_{1: 3}^{-1}$ and $\mathcal{M}_{23}^{-1}$ are independent of each other; from the four independent mixed Higgs-boson propagators, two are CP violating.

\section{Superweak-like model with two Higgs doublets}

Usually, the symmetry $S$ is applied to the quark sector in the following way: the lefthanded-quark doublets $q_{L}$ are invariant under $S$ and, either both the $p_{R}$ and the $n_{R}$ are invariant under $S$, or the $p_{R}$ are invariant but the $n_{R}$ transform to $-n_{R}$ under $S$. In both cases the symmetry $S$ leads to the absence of FCNI, which is precisely what one usually wants from it. In the paper of Branco and Rebelo [9], the role of the symmetry $S$ was different: it was meant there to provide a rationale for the smallness of the CP. violating FCNI as compared to the CP-conserving FCNI; those authors applied the symmetry $S$ to the quark sector in a more-or-less arbitrary way, allowing for the FCNI, because they needed these in order to explain the observed CP noninvariance. In the model that I will now present, the symmetry $S$ has, besides this useful role of allowing for a natural suppression of $C P$ violation, still another one, and more fundamental role: I use it to avoid that, though $\mathrm{CP}$ is spontaneously broken - a relative phase $\alpha$ between the vevs appears - the mass matrices become complex. On the other hand, as there is no need for CP-violating FCNI in the up-quark sector, I will also use $S$ to eliminate the FCNI in that sector (though this latter feature of the model is not essential). I assume that the doublets $q_{L}$, the right-handed quarks $p_{R}$, and two of the three right-handed quarks $n_{R}, n_{R 1}$ and $n_{R 2}$, are invariant under $S$; but

$$
S: n_{l i 3} \rightarrow-n_{R 3}
$$

As a consequence of these assignments, the matrix $\Delta_{2}$ vanishes, and therefore $N_{u}=$ $\left(v_{2} / v_{1}\right) D_{u}$, which means that there are no FCNI in the up-quark sector. On the other hand, the matrices $\Gamma_{1}$ and $\Gamma_{2}$ have the form

$$
\Gamma_{1} \sim\left(\begin{array}{ccc}
x & x & 0 \\
x & x & 0 \\
x & x & 0
\end{array}\right), \quad \Gamma_{2} \sim\left(\begin{array}{ccc}
0 & 0 & x \\
0 & 0 & x \\
0 & 0 & x
\end{array}\right),
$$

where the crosses denote non-zero matrix elements. The mass matrix of the down-type quarks. $\left[v_{1} \Gamma_{1}+v_{2} \exp (i \alpha) \Gamma_{2}\right] / \sqrt{2}$, has its first two columns real, while the whole third column has a common phase $\alpha$. But that phase may be eliminated by a rephasing of $n_{R: 3}, n_{R: 3} \rightarrow \exp (-i \alpha) n_{R: 3}$, after which rephasing the mass matrix becomes real. As a consequence, the diagonalising matrices $U_{n l}, U_{n k}, U_{p L}$ and $U_{p R}$ are all real and orthogonal, and the CKM matrix $V$ is real. The matrices $N_{u}$ and $N_{d}$ are also real after the rephasing of $n_{R: 3}$, and therefore there is CP violation neither via $W^{ \pm}$exchange nor via $H^{ \pm}$exchange. The couplings of $H^{\cup}$ and of $R$ are real and the ones of $I$ are purely 
imaginary, and all the CP violation originates in the mixing of $I$ with $H^{\prime \prime}$ and $R$. The model is superweak-like.

I now derive the form of the matrix $N_{d}$. I start from Eq. 8. I define

$$
K:=\frac{v_{2}}{v_{1}}+\frac{v_{1}}{v_{2}}=\frac{v^{2}}{v_{1} v_{2}} \geq 2
$$

I also define $\Gamma_{1.2}^{\prime}:=U_{n L}^{\dagger} \Gamma_{1.2}$. We therefore have, after the phase $\alpha$ has been removed by the rephasing of $n_{R: 3}$,

$$
\begin{aligned}
& \frac{v_{1}}{\sqrt{2}} \Gamma_{1}^{\prime}+\frac{v_{2}}{\sqrt{2}} \Gamma_{2}^{\prime}=D_{d} U_{n R}^{\dagger}, \\
& N_{d}=\frac{v_{2}}{v_{1}} D_{d}-K \frac{v_{2}}{\sqrt{2}} \Gamma_{2}^{\prime} U_{n R} .
\end{aligned}
$$

The important point is that the matrices $\Gamma_{1}^{\prime}$ and $\Gamma_{2}^{\prime}$ have exactly the same form than the matrices $\Gamma_{1}$ and $\Gamma_{2}$, respectively (see Eq. 25). Therefore, if the third column of the orthogonal matrix $U_{n R}^{\dagger}$ is denoted by $(x, y, z)^{T}$, with

$$
x^{2}+y^{2}+z^{2}=1
$$

Eq. 27 tells us that the third column of $\Gamma_{2}$ is $\left(\sqrt{2} / v_{2}\right)\left(m_{d} x, m_{s} y, m_{b} z\right)^{T}$, the other two columns being of course zero. Eq. 28 then gives

$$
N_{d}=\left(\begin{array}{ccc}
m_{d}\left(\frac{v_{2}}{v_{1}}-K x^{2}\right) & -m_{d} K x y & -m_{d} K x z \\
-m_{a} K x y & m_{*}\left(\frac{v_{2}}{v_{1}}-K y^{2}\right) & -m_{1} K y z \\
-m_{b} K x z & -m_{b} K y z & m_{b}\left(\frac{w_{2}}{v_{l}}-K z^{2}\right)
\end{array}\right) .
$$

Thus, the strength of the flavor-changing couplings in this model is parametrized by three real numbers of modulus not greater than $1 / 2$,

$$
\beta_{k}:=x y, \quad \beta_{B_{d}}:=x z, \quad \beta_{B_{s}}:=y z,
$$

which satisfy

$$
\beta_{K} \beta_{B_{d}} \beta_{B_{s}}=\left(\beta_{k} \beta_{B_{d}}\right)^{2}+\left(\beta_{K} \beta_{B}\right)^{2}+\left(\beta_{B_{d}} \beta_{B_{s}}\right)^{2}
$$

An important question concerning superweak models is: how does the strength of the superweak interaction scale from the kaon system to the other neutral-meson systems? Some tentative answers to this question have been suggested in the past [10], like a scaling with either the square-root of the masses, or with the masses themselves. of the intervenient quarks; but these suggestions were never based on complete superweak or superweak-like models, and they were only educated guesses. In my model, we observe in Eq. 30 that there is certainly a scaling of the FCNI with the quark masses, but that this scaling may be irrelevant, because the three $\beta$ parameters may be quite different. For instance, if we assume that two of the three $\beta$ parameters vanish, the third one then remains completely arbitrary (see Eq. 32). Thus, the fact that $\beta_{k}$ has a certain value does not allow us to predict the value of $\beta_{B_{t}}$, and with it the strength of the superweak interaction in the $B_{d}$ system. But, if two of the $\beta$ parameters happened to be known, we could, from Eq. 32 , predict the value of the third one. 
A further interesting point is that the overall str'ngth of the superweak interactions, which is given by $K$, is inversely proportional to $t$ if one of the vevs decreases. The off-diagonal between the bi-diagonalization of the mass matr They therefore increase when one of the Yukawa mass matrix, and therefore determines more strol to be followed. If for instance $v_{1} \gg v_{2}$, then the $1:$ mass matrix much greater than the one of $\Gamma_{2}$, anc. the diagonalization matrices $U_{n l}$. and $U_{n / R}$.

Let us calculate the contribution to the neutr: from the neutral-Higgs-exchange diagram in Figure oduct $\left(v_{1} v_{2}\right)$, and thus increases ints of $N_{d}$ express a mismatch $d$ the one of either $\Gamma_{2}$ or of $\Gamma_{1}$. ices has a greater weight in the he bi-diagonalization procedure ix $\Gamma_{1}$ gives a contribution to the erefore $\Gamma_{1}$ essentially determines

aon mixing-matrix element $M_{12}$ The interaction of the three real neutral Higgs fields with the strange and down qua ks is given by the following terms in the Lagrangian:

$$
\begin{aligned}
& \left(K \beta_{k} / 2 v\right) \sum_{j=1}^{3} H_{j}\left\{\overline { s } \left[T_{2 j}\left(m_{*}+m_{t l}\right)+i T_{3 j}\left(m_{s}-m_{t l}\right)\right.\right. \\
& \left.\left.+T_{2 j}\left(m_{*}-m_{t l}\right) \gamma_{s i}+i T_{3 j}\left(m_{*}+m_{t l}\right) \gamma_{s}\right] d+\text { h.c. }\right\}
\end{aligned}
$$

where the matrix $T$ was introduced in Eq. 21. Therefcre, the diagram of Fig. 1 gives

$$
\begin{aligned}
& \mathcal{H}_{+1 f}=-\left(K \beta_{k} / 4 v\right)^{2} \\
& \times\left\{(\bar{s} d)^{2}\left[\left(m_{a}+m_{d l}\right)^{2}\left(\mathcal{M}^{-1}\right)_{22}-\left(m_{a}-m_{d}\right)^{2}\left(\mathcal{M}^{-1}\right)_{33}+2 i\left(m_{s}^{2}-m_{d}^{2}\right)\left(\mathcal{M}^{-1}\right)_{23}\right]\right. \\
& \left.+\left(\bar{s} \gamma_{\cdot ;} d\right)^{2}\left[\left(m_{a}-m_{d}\right)^{2}\left(\mathcal{M}^{-1}\right)_{22}-\left(m_{a}+m_{d}\right)^{2}\left(\mathcal{M}^{-1}\right)_{3: 3}+2 i\left(m_{s}^{2}-m_{d}^{2}\right)\left(\mathcal{M}^{-1}\right)_{2: 3}\right]\right\}
\end{aligned}
$$

Using the fact that $m_{*} \gg m_{d}$, and the vacuum-insertion value for the matrix element [11]

$$
\left\langle\overline{K^{\prime \prime}}\left|(\bar{s} d)^{2}+\left(\bar{s} \gamma_{5} d\right)^{2}\right| K^{-u}\right\rangle=\frac{5}{6} \frac{f_{k}^{2} \cdot m_{k}^{3}}{m_{s}^{2}}
$$

one obtains

$$
M_{12}^{\text {llikgs }} \approx-\frac{5}{96} K^{2} \beta_{K}^{2} \frac{f_{k}^{2} \cdot m_{k}^{3}}{v^{2}}\left[\left(\mathcal{M}^{-1}\right)_{22}-\left(\mathcal{M}^{-1}\right)_{33}+2 i\left(\mathcal{M}^{-1}\right)_{23}\right]
$$

From the phenomenology of the neutral kaons, we know that $\left|\operatorname{Re} M_{12}\right| \approx \Delta m_{k} / 2$ and $\left|\operatorname{Im} M_{12}\right| \approx \sqrt{2}|\epsilon| \Delta m_{K}$. Assuming the product $\left(K \beta_{K}\right)$ to be of order 1, one therefore obtains

$$
\begin{gathered}
\left.\mid i \cdot \mathcal{M}^{-1}\right)_{233} \mid \approx 0.002 \mathrm{TeV}^{-2} \\
\left|\left(\mathcal{M}^{-1}\right)_{22}-\left(\mathcal{M}^{-1}\right)_{333}\right|<0.65 \mathrm{TeV}^{-2}
\end{gathered}
$$

Eq. 38 is only an approximate upper bound, because there are other contributions to the real part of $M_{12}$, as the box-diagram contributions and a long-distance contribution; but $\mathrm{Eq} .37$ is an equality, because the diagram of Fig. 1 really gives, in our model, the dominant contribution to $\operatorname{Im} M_{12}$.

Naively, Eq. 37 would give a lower bound for the masses of the Higgs bosons of the order of $10 \mathrm{TeV}$, stronger than the bound from Eq. 38. But as $\mathcal{M}_{23}^{-1}$ is proportional to 
$\cot \alpha$, and this parameter may naturally be set to be very small, say $\sim 10^{-3}$, this is not really a problem. On the other hand, it is obviously difficult to conciliate Eq. 38 with the non-existence of Higgs bosons with masses of the order of the $\mathrm{TeV}$. One possibility consists in assuming a rather strong cancellation between $\left(\mathcal{M}^{-1}\right)_{22}$ and $(\mathcal{M})_{33}^{-1}$, which of course requires a certain amount of fine-tuning. Also note that $H^{\prime \prime}$ may always be an approximate eigenstate of the propagation, and may then have an arbitrarily small mass. Only $R$ and $I$ are required to be heavy, or else to have very similar masses, so that their contributions cancel. There is of course also the possibility of obtaining smaller masses for the Higgs bosons, by assuming $M_{12}^{\text {likxs }}$ to be suppressed by a very small $\beta_{K}$ instead. But this is dangerous: while $M_{12}^{H \text { Higgs }}$ is proportional to $\beta_{K}^{2}$, because of the existence in the diagram of Fig. 1 of two flavor-changing vertices, the Higgsexchange diagrams giving an imaginary part to the kaon decay amplitudes (Figure 2) have only one flavor-changing vertex, and are therefore proportional only to $\beta_{K}$. Therefore, a low $\beta_{k}$ enhances $\epsilon^{\prime} / \epsilon$, and the superweak character of the model may thus be lost.

Still concerning the superweak character of this model, it must be remarked that, while the imaginary part of $M_{12}$ is proportional to $\mathcal{M}_{23}^{-1}$, the imaginary parts of the decay amplitudes also involve $\mathcal{M}_{1: 3}^{-1}$, because the field $H^{0}$, instead of the field $R$, may be present in the flavor-diagonal vertex in Fig. 2. Therefore, another possibility for enhancing $\epsilon^{\prime} / \epsilon$ is considering $\mathcal{M}_{1: 3}^{-1}$ to be some two orders of magnitude [5] larger than $\mathcal{M}_{23}^{-1}$. This possibility is realized, in particular, if we require $H^{\cup}$ to be an approximate eigenstate of the mass matrix, with a mass much smaller than the ones of the other two eigenstates. For these reasons, it cannot be claimed that the present model is automatically and in all cases superweak in nature.

A remarkable fact about Eq. 36 is the proportionality of $M_{12}^{\text {lliggs }}$ to $\left[\left(\mathcal{M}^{-1}\right)_{22}-\right.$ $\left.\left(\mathcal{M}^{-1}\right)_{33}+2 i\left(\mathcal{M}^{-1}\right)_{23}\right]$. This proportionality hold; because $m_{d} \ll m_{s}$, and is of course also valid if the external quarks in the diagram were, instead of $s$ and $d$, say, $b$ and $d$. This means that the neutral-Higgs-exchange contributions to $M_{12, K}, M_{12, B_{d}}$ and $M_{12 . B}$, have all the same phase. Of course, the three $M_{12}$ themselves do not need to have the same phase, because they also receive other contributions, for instance, from $W^{ \pm}$and $H^{ \pm}$box diagrams. Those further contributions are, however, all real. In a certain sense, we thus have here the situation exactly opposite to the one which was studied by Soares and Wolfenstein [3]: the superweak contribution to $M_{12}$ has a common and non-zero phase, while the standard-model and all other contributions are real.

\section{More than two Higgs doublets}

When one tries to extend the ideas of the last section to models with $n>2$ Higgs doublets, one meets the following problem: how to avoid a complex mixing among the charged Higgs bosons, leading to CP violation in the exchange of these? Let us first understand the origin of this problem. Suppose that, by using a symmetry as in the last section, we are able to enforce mass matrices for the quarks which may be made real by an adequate rephasing of the quark fields. Then, after that rephasing, the 
lukawa couplings of the $n$ fields $\varphi_{j}^{+}, j$ from 1 to $n$, will also be real. Now, from gauge invariance we know that the field $\sum_{j=1}^{n} v_{j} \varphi_{j}^{+}$is the charged Goldstone boson $G^{+}$. The $n-1$ charged Higgs fields orthogonal to $G^{+}$are free to mix. If $n=2$, there is only one charged Higgs field orthogonal to $\mathrm{G}^{+}$, and no mixing among charged Higgs fields is therefore possible. But if $n \geq 3$ mixing occurs, and in principle, because of the relative phases of the vevs, that mixing is complex. This is indeed the main mechanism of $\mathrm{CP}$ violation in the Weinberg model [12]. The complex mixing of the charged Higgs fields leads to complex Yukawa couplings of the physical bosons. I want to avoid this mechanism of $\mathrm{CP}$ violation.

In order to achieve this, the idea is to have a spontaneous CP violation which, in some way, only involves effectively two of the $n$ Higgs doublets, such that the mixing of the Higgs fields is cnmplex only in a two-doublet sector. As an instance of the general method by which this idea can be realized, suppose that there are four Higgs doublets, and that the Higgs potential is

$$
\begin{aligned}
\mathcal{L}_{l 1}= & \sum_{j=1}^{\dagger}\left[\mu_{j} H_{j}^{\dagger} H_{j}+\lambda_{j}\left(H_{j}^{\dagger} H_{j}\right)^{2}\right] \\
& +\sum_{j<k}\left[\chi_{j k}\left(H_{j}^{\dagger} H_{j}\right)\left(H_{k}^{\dagger} H_{k}\right)+\theta_{j k}\left(H_{j}^{\dagger} H_{k}\right)\left(H_{k}^{\dagger} H_{j}\right)\right] \\
& x\left[\left(H_{1}^{\dagger} H_{1}\right)^{2}+\left(H_{1}^{\dagger} H_{1}\right)^{2}\right]+\nu\left(H_{1}^{\dagger} H_{4}+H_{4}^{\dagger} H_{1}\right) \\
& +y\left[\left(H_{1}^{\dagger} H_{3}\right)\left(H_{1}^{\dagger} H_{3}\right)+\left(H_{3}^{\dagger} H_{1}\right)\left(H_{3}^{\dagger} H_{4}\right)\right] \\
& +z\left[\left(H_{1}^{\dagger} H_{2}\right)\left(H_{3}^{\dagger} H_{2}\right)+\left(H_{2}^{\dagger} H_{1}\right)\left(H_{2}^{\dagger} H_{3}\right)\right]
\end{aligned}
$$

All the coefficients are real because of CP invariance. There are four terms in this potential which can "see" the three relative phases of the four vevs, and therefore spontaneous CP violation is possible. We notice however that there is only one relative phase between vevs, the one between the vevs of $H_{1}^{\prime \prime}$ and of $H_{*}^{\prime \prime}$, which is "seen" by more than one term in the potential, the terms with coefficients $x$ and $\nu$. Once the clash between these two terms leads to a non-trivial phase between the vevs of $H_{1}^{\prime \prime}$ and of $H_{1}^{\prime}$, the terms with coefficients $y$ and $z$ lead to phases for the vevs of $H_{3}^{\prime}$ and of $H_{2}^{\prime \prime}$, respectively, which simply cancel the non-trivial phase difference generated in the $H_{1}^{\prime \prime}-H_{1}^{\prime \prime}$ sector. As the spontaneous CP breaking is generated by only $\imath$ wo Higgs doublets. the complexities in the mixing of the Higgs fields also remain limited to the mixing of those two doublets, and no complex mixing of charged Higgs fields can arise. Notice however that the terms with coefficients $y$ and $z$, though they are neutral in what concerns the CP breaking, are needed, because without them one would have undesirable Goldstone bosons in the neutral sector, due to the possibility of independent rephasings of the doublets $H_{3}$ and $H_{2}$.

A question arises: is the potential in Eq. 39 stable under radiative corrections? It is. That potential possesses a symmetry $S^{\prime \prime}$ of the type $Z_{x}$, under which, with $\omega^{8}=1$ but $\omega=1$,

$$
H_{1} \rightarrow H_{1}, \quad H_{2} \rightarrow \omega H_{2}, \quad H_{3} \rightarrow \omega^{2} H_{3}, \quad H_{4} \rightarrow \omega^{1} H_{4} .
$$


This symmetry $Z_{R}$ is softly broken by the term with coefficient $\nu$ to the symmetry $Z_{1}$ obtained by identifying $\omega^{\prime}$ with 1 . The symmetry $Z_{\star}$ forbids the appearance of divergent contributions to other quartic terins in the potential, and the symmetry $Z_{\text {I }}$ forbids the appearance of any further soft-breaking terms, so that the whole potential is stable.

If we write the Higgs doublets as

$$
H_{j}=e^{i \alpha_{j}}\left(\begin{array}{c}
\varphi_{j}^{+} \\
\frac{1}{\sqrt{2}}\left(v_{j}+\rho_{j}+i \eta_{j}\right)
\end{array}\right),
$$

then we find the following stability equations which determine the relative phases of the vevs:

$$
\begin{aligned}
-2 x v_{1} v_{1} \cos \left(\alpha_{1}-\alpha_{1}\right) & =\nu, \\
y \cos \left(2 \alpha_{3}-\alpha_{1}-\alpha_{4}\right) & =-|y|, \\
z \cos \left(2 \alpha_{2}-\alpha_{1}-\alpha_{3}\right) & =-|z| .
\end{aligned}
$$

The last two of these equations embody what was said above, that $\alpha_{3}$ and $\alpha_{2}$ adjust in order to offset the effects of the spontaneous CP breaking, which occurs in the sector $H_{1}^{\prime \prime}-H_{1}^{\prime \prime}$. After a bit of work one obtains the mass matrices of both the charged and the neutral Higgs fields. The one of the charged Higgs fields is the most general real mass matrix compatible with the requirement that it has an eigenvector $\sum_{j=1}^{+} v_{j} \varphi_{j}^{+}$with zero eigenvalue, and therefore it is essentially uninteresting. In the neutral sector, we have the following. First, a mass matrix for the four fields $\rho_{j}$, which has no constraints on it, and therefore is not worth writing down explicitly. For the four fields $\eta_{j}$ one has the mass terms

$$
\begin{aligned}
& x \sin ^{2}\left(\alpha_{1}-\alpha_{4}\right) v_{1}^{2} v_{1}^{2}\left(\frac{\eta_{1}}{v_{1}}-\frac{\eta_{1}}{v_{4}}\right)^{2} \\
& +\left(|y| v_{1} v_{3}^{2} v_{4} / 4\right)\left(\frac{\eta_{1}}{v_{1}}+\frac{\eta_{4}}{v_{4}}-2 \frac{\eta_{3}}{v_{3}}\right)^{2} \\
& +\left(|z| v_{1} v_{2}^{2} v_{3} / 4\right)\left(\frac{\eta_{11}}{v_{1}}+\frac{\eta_{3}}{v_{3}}-2 \frac{\eta_{2}}{v_{2}}\right)^{2} .
\end{aligned}
$$

Notice the way in which the coefficients $y$ and $z$ kill two menacing Goldstone bosons. Unfortunately, the three fields in Eq. 45 can not be interpreted as mass eigenstates, because they are not mutually orthogonal. Finally, there is only one term which mixes the $\rho_{j}$ components with the $\eta_{j}$ components, and which is the source of CP violation:

$$
x \sin \left[2\left(\alpha_{1}-a_{1}\right)\right]\left(v_{1} \eta_{4}-v_{1} \eta_{1}\right)\left(v_{1} \rho_{4}+v_{4} \rho_{1}\right) .
$$

As expected, this term only involves the neutral components of $H_{1}$ and of $H_{4}$.

It is clear that the mechanism worked out in this section for the case of four Higgs doublets may be applied in general for any number $n$ of Higgs doublets. One then uses a discrete symmetry $S^{\prime}$ of the type $Z_{2 n-1}$, softly broken to a symmetry $Z_{2 n-2}$ by one term in the Higgs potential. CP is spontaneously broken only in a two-Higgs-doublets sector, in which one of the doublets is invariant under $S^{\prime}$ and the other one transforms with a minus sign under $S^{\prime}$. The other Higgs doublets transform under $S^{\prime}$ in such a way as to allow, for each of them, one and only one term in the potential which "sees" its phase, in order to kill the corresponding Goldstone boson. The whole mechanism is clearly a generalization of the two-doublet case worked out in section 2 . 


\section{Superweak-like model with four Higgs doublets}

I now use the insights of the previous section to construct, as a practical example, a superweak-like model with four Higgs doublets.

The way in which the symmetry $S^{\prime}$ acts on the four Higgs doublets was given in Eq. 40. I take the quark multiplets to transform under $S^{\prime}$ as

$$
\begin{array}{cc}
\overline{q_{I .1}} \rightarrow \overline{q_{L 1}}, \quad \overline{q_{L 2}} \rightarrow \omega \overline{q_{I .2}}, \quad \overline{q_{I .3}} \rightarrow \omega^{2} \overline{q_{L 3}} \\
p_{R 1} \rightarrow \omega^{1} p_{R 1}, \quad p_{R 2} \rightarrow \omega^{3} p_{R 2}, \quad p_{R 3} \rightarrow \omega^{6} p_{R 3}, \\
n_{R 1} \rightarrow \omega^{3} n_{R 1}, \quad n_{R 2} \rightarrow \omega^{1} n_{R 2}, \quad n_{R 3} \rightarrow \omega^{5} n_{R 3} .
\end{array}
$$

Then, the Yukawa Lagrangian is

$$
\begin{aligned}
-\mathcal{L}_{1}= & a \overline{q_{L 1}} H_{1} n_{R 2}+b \overline{q_{L 2}} H_{1} n_{R 1} \\
& +c \overline{q_{L 2}} H_{3} n_{R 3}+d \overline{q_{1,3}} H_{3} n_{R 2}+e \overline{q_{L 3}} H_{2} n_{R 3} \\
& +f \overline{q_{L 1}}\left(i \sigma_{2} H_{1}^{*}\right) p_{R 1}+g \overline{q_{L 2}}\left(i \sigma_{2} H_{1}^{*}\right) p_{R 2} \\
& +h \overline{q_{L 3}}\left(i \sigma_{2} H_{1}^{*}\right) p_{R 3}+\text { h.c. }
\end{aligned}
$$

The coupling constants are real. When the Higgs doublets acquire vevs, one obtains a. diagonal mass matrix for the up-type quarks,

$$
m_{u}=|f| \frac{v_{4}}{\sqrt{2}}, \quad m_{c}=|g| \frac{v_{4}}{\sqrt{2}}, \quad m_{t}=|h| \frac{v_{1}}{\sqrt{2}},
$$

and a mass matrix for the down-type quarks of the form

$$
\left(\begin{array}{ccc}
0 & \times & 0 \\
\times & 0 & \times \\
0 & \times & \times
\end{array}\right) .
$$

The arbitrary phases in a quark mass matrix of this form may be rotated away by means of rephasings of the quark fields, so that one may take, for the purpose of the analysis of the Yukawa couplings, all the vevs to be real. The advantage of having a down-quark mass matrix of the form in Eq. 50, when the mass matrix of the up-type quarks is diagonal, is that one immediately obtains a constraint on the quark masses and the parameters of the CKM matrix which works particularly well (13):

$$
\left(M_{11} M_{d}^{\dagger}\right)_{12}=m_{d}^{2} V_{11} V_{21}^{*}+m_{s}^{2} V_{12} V_{22}^{*}+m_{b}^{2} V_{13} V_{23}^{*}=0
$$

in which $V$ is the CKM matrix, which is in this case real and orthogonal. This leads to a prediction for $\left|V_{u b} / V_{c b}\right|$ as a function of $\left|V_{u s}\right|$, of $\left|V_{c b}\right|$ and of $\delta:=\left(m_{s}^{2}-m_{d}^{2}\right) /\left(m_{b}^{2}-m_{d}^{2}\right)$ :

$$
\begin{aligned}
& U_{r b b}=\left(1 / 2 U_{c b}\right)\left(U_{c b}-2 \delta U_{u s} U_{r b}+\delta^{2} U_{u s}\right. \\
& \left.-\sqrt{U_{c b}^{2}-4 \delta U_{u s} U_{c b}^{2}-2 \delta^{2} U_{u s} U_{c b}\left(1-2 U_{u s}-2 U_{c b}\right)-4 \delta^{3} U_{u s}^{2} U_{c b}+\delta^{1} U_{u s}^{2}}\right),
\end{aligned}
$$


where $L_{1}:=\left|V_{1}\right|^{2}$. This prediction agrees very well with experiment, at least if we use for $m$. the central value $175 \mathrm{MeV}$ [14]. It should be pointed out that mass matrices of the type in Eq. 50 may be obtained in a simpler way by means of a $Z_{6}$ symmetry in a model with only three Higgs doublets [13]; but that simpler implementation is not good for our purposes, because in that case one would obtain CP violation via the exchange of charged Higgs fields.

The model suggested in this section has, over the one of section 3 , the advantage that it has, except for the ratios of the four vevs, $v_{1} \div v_{3} \div v_{2} \div v_{1}$, no unknown parameters in the matrices of Yukawa couplings of the Higgs fields. There are here no annoying parameters like the $\beta$ parameters of section 3 . However, the more Higgs doublets there are, the more unknown independent parameters arise in the mixed propagators of the Higgs fields (the matrix element; of $\mathcal{M}^{-1}$ ). Indeed, it can be shown that models with a Higgs potential of the type studied in section 4 , have $\left(n^{2}+3 n-2\right) / 2$ independent neutral-Higgs mixed propagators, out of which $n$ propagators are CP-violating $(n \geq 2$ is the number of Higgs doublets). Therefore, the present four-Higgs-doublet model has really no more predictive power in what concerns $C P$ violation than the two-Higgsdoublet model given in section 3 .

\section{Conclusions}

The main conclusions of this work are the following.

It is possible to construct extensions of the standard model, with $n \geq 2$ Higgs doublets. in which CP violation occurs only in the propagators of the neutral Higgs fields, via scalar-pseudoscalar mixing, and there is neither Kobayashi-Maskawa CP violation. nor $C P$ violation via the exchange of charged Higgs bosons. The neutral Higgs bosons have flavor-changing Yukawa couplings, and their exchange in a treelevel diagram explains the observed CP violation. The essential ingredients of these models are:

1) CP invariance at the Lagrangian level, enforcing real Yukawa coupling matrices;

2) a discrete symmetry $Z_{2^{n-1}}$, which constrains the Yukawa coupling matrices in such a way, that the phases which the vevs feed into the quark mass matrices may be absorbed in the quark fields, the mass matrices thus becoming real;

3) the discrete symmetry also constrains the Higgs potential in such a way that it has exactly as many terms which see the relative phases of the doublets, as there are relative phases, which fact avoids the appearance of Goldstone bosons;

4) one term in the Higgs potential which breaks the discrete symmetry softly to $Z_{2^{n-2}}$, allowing for spontaneous CP violation to occur.

The discrete symmetry may be chosen to transform the fields in such a way that there are no neutral flavor-changing Yukawa interactions in the up-type-quark sector. This feature of the models that $I$ have presented may however be altered, if experiment should dictate it.

There are three types of unknown parameters which affect our ability to make predictions from such models: 
1) the ratios of the vevs;

2) the values of the mixed propagators of the neutral Higgs fields;

3) unknown real parameters in the Yukawa couplings.

The unknown parameters of the first two types increase in number with the number $n$ of Higgs doublets. Therefore, a model with two Higgs doublets has advantages. I have worked out one such model in particular detail. Even in that very simple case, there are no clear predictions for the way in which the superweak interaction responsible for the observed CP violation should scale to the neutral- $B$-meson systems.

I thank L. Wolfenstein for illuminating discussions. Both he and L.-F. Li have read and criticised the manuscript. This work was supported by the United States Department of Energy, under the contract DE-FG02-91ER-40682.

\section{References}

(1) L. Wolfenstein, Phys. Rev. Let̂. 13, 562 (1964).

[2] B. Winstein, Phys. Rev. Lett. 68, 1271 (1992);

J. M. Soares and L. Wolfenstein, Carnegie-Mellon report CMU-HEP92-01 (1992), to be published in Phys. Rev. D.

[3] J. M. Soares and L. Wolfenstein, Carnegie-Mellon report CMU-HEP92-11 (1992), unpublished.

[4] G. Buchalla, A. J. Buras and M. K. Harlander, Nucl. Phys. B 337, 313 (1990).

See also:

J. Fröhlich, J. Heinrich, E. A. Paschos and J.-M. Schwarz, Dortmund report DOTH-91-02 (1991), unpublished;

J. Heinrich, E. A. Paschos, J.-M. Schwarz and Y.-L. Wu, Phys. Lett. B 279, 140 (1992).

[5] J. Liu and L. Wolfenstein, Nucl. Phys. B 289, 1 (1987).

[6] M. Kobayashi and T. Maskawa, Prog. Theor. Phys. 49, 652 (1973).

[7] T. D. Lee, Phys. Rev. D 8, 1226 (1973).

(8) C. D. Froggatt, R. G. Moorhouse and I. G. Knowles, Argonne report ANL-HEPPR-92-01 (1992), unpublished.

19] G. C. Branco and M. N. Rebelo, Phys. Lett. B 160, 117 (1985).

[10] T.-P. Cheng and M. Sher, Phys. Rev. D 35, 3484 (1987);

J. Liu and L. Wolfenstein, Phys. Lett. B 197, 536 (1987);

J.-N. Gérard and T. Nakada, Phys. Lett. B 261, 474 (1991). 
(11] B. McWilliams and O. Shanker, Phys. Rev. D 22, 2853 (1980).

[12] S. Weinberg, Phys. Rev. Lett. 37, 657 (1976);

G. C. Branco, Phys. Rev. D 22, 2901 (1980).

[13] E. Ma and D. Ng, Phys. Rev. Lett. 65, 2499 (1990);

E. Ma and K. Mcllhany, Mod. Phys. Lett. A 6, 1089 (1991).

[14] J. Gasser and H. Leutwyler, Phys. Rep. 87, 77 (1983).

\section{DISCLAIMER}

\footnotetext{
This report was prepared as an account of work sponsored by an agency of the United States Government. Neither the United States Gnvernment nor any agency thereof, nor any of their employees, makes any warranty, express or implied, or assumes any legal liability or responsibility for the accuracy, completeness, or usefulness of any information, apparatus, product, or process disclosed, or represents that its use would not infringe privately owned rights. Reference herein to any specific commercial product, process, or service by trade name, trademark, manufacturer, or otherwise does not necessarily constitute or imply its endorsement, recommendation, or favoring by the United States Government or any agency thereof. The views and opinions of authors expressed herein do not necessarily state or reflect those of the United States Government or any agency thereof.
} 


\section{FIGURE CAPTIONS}

Figure 1: Higgs-exchange diagram giving an imaginary part to the neutral-kaon mixing matrix element $M_{12}$, and therefore leading to a non-zero $\epsilon$.

Figure 2: Higgs-exchange diagrams giving imaginary parts to the $K^{\prime \prime} \rightarrow 2 \pi$ decay matrix elements $A_{2}$ and $A_{11}$, and therefore leading to a non-zero $\epsilon^{\prime}$. 


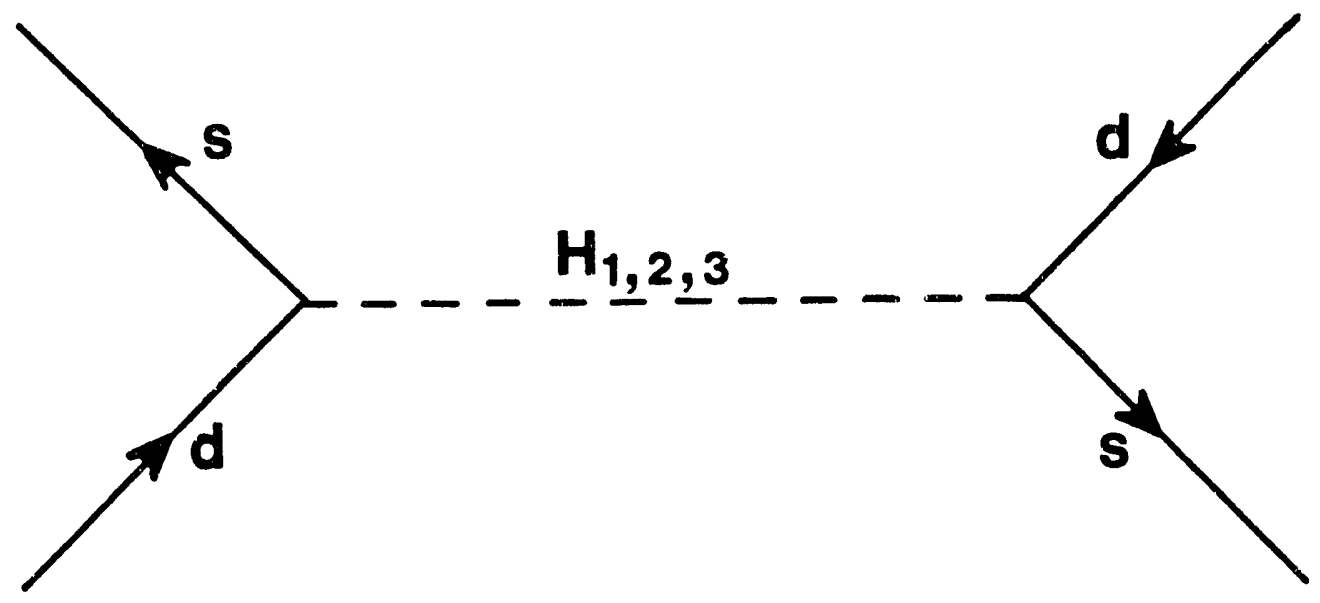

Fig.1 


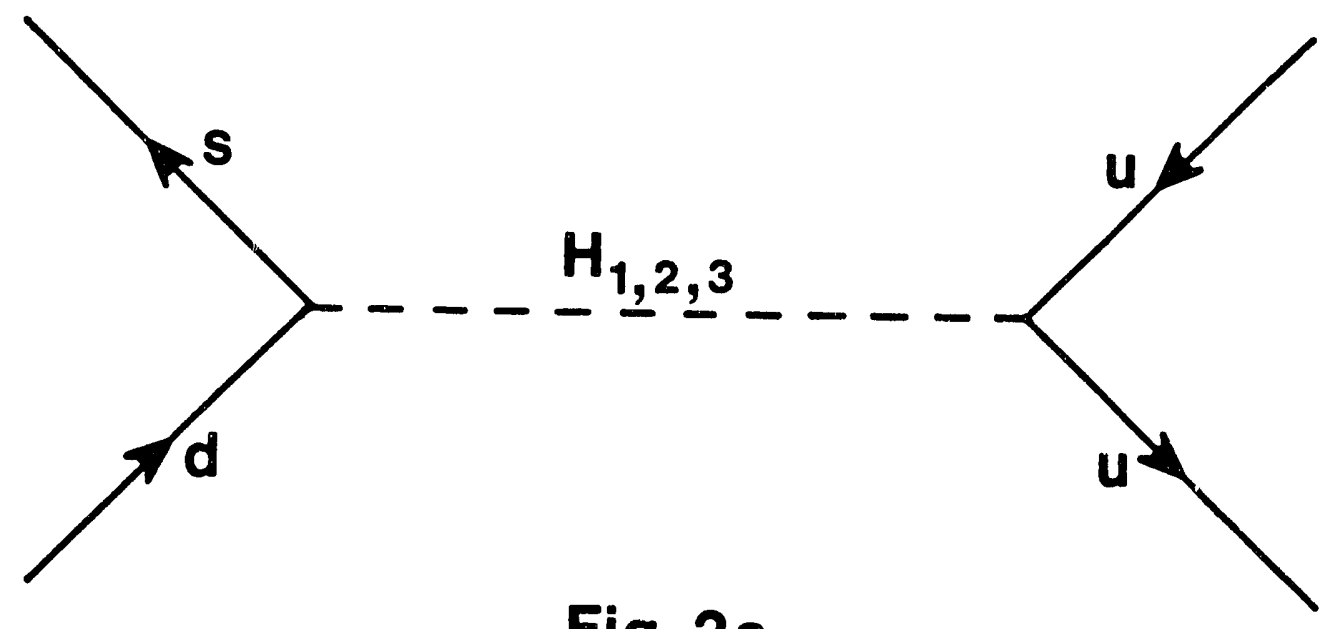

Fig. 2a

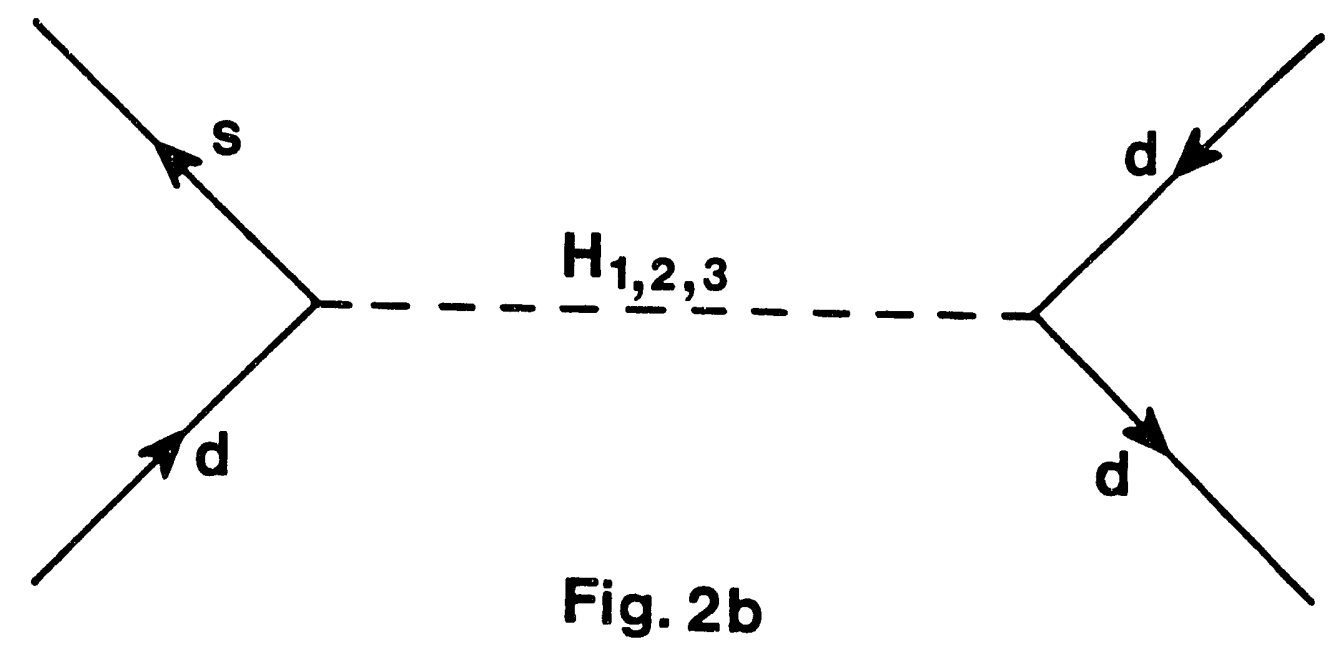



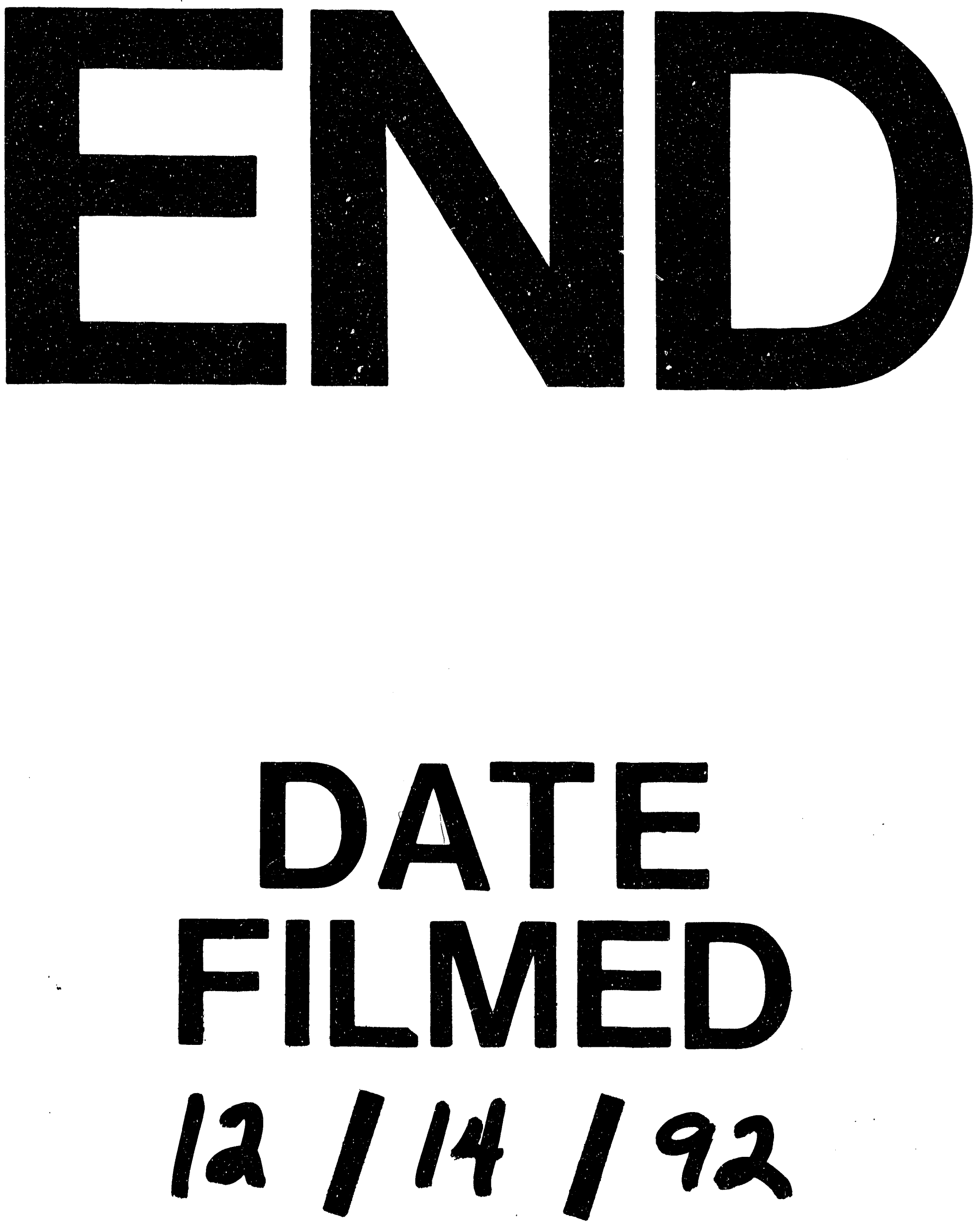

92 
